\title{
Signalling plasticity and energy saving in a tropical bushcricket
}

\author{
M. Hartbauer $\cdot$ A. Stabentheiner $\cdot$ H. Römer
}

Received: 7 September 2011/Revised: 27 October 2011/Accepted: 3 November 2011/Published online: 18 November 2011

(C) The Author(s) 2011. This article is published with open access at Springerlink.com

\begin{abstract}
Males of the tropical bushcricket Mecopoda elongata synchronize their acoustic advertisement signals (chirps) in interactions with other males. However, synchrony is not perfect and distinct leader and follower roles are often maintained. In entrainment experiments in which conspecific signals were presented at various rates, chirps displayed as follower showed notable signal plasticity. Follower chirps were shortened by reducing the number and duration of syllables, especially those of low and medium amplitude. The degree of shortening depended on the time delay between leader and follower signals and the sound level of the entraining stimulus. The same signal plasticity was evident in male duets, with the effect that the last syllables of highest amplitude overlapped more strongly. Respiratory measurements showed that solo singing males producing higher chirp rates suffered from higher metabolic costs compared to males singing at lower rates. In contrast, respiratory rate was rather constant during a synchronous entrainment to a conspecific signal repeated at various rates. This allowed males to maintain a steady duty cycle, associated with a constant metabolic rate. Results are discussed with respect to the preference for leader signals in females and the possible benefits males may gain by overlapping their follower signals in a chorus.
\end{abstract}

Keywords Signal timing - Signal plasticity · Calling metabolism $\cdot$ Chorus synchrony .

Acoustic communication

M. Hartbauer $(\bowtie) \cdot$ A. Stabentheiner $\cdot$ H. Römer

Department of Zoology, Karl-Franzens Universität Graz,

Graz, Austria

e-mail: manfred.hartbauer@uni-graz.at

\author{
Abbreviations \\ SPL Sound pressure level \\ CP Chirp period
}

\section{Introduction}

One function of acoustic signals is to attract a mating partner from some distance; they are often displayed in a highly repetitive and redundant manner. The energetic costs associated with such ongoing displays have been studied in animals with highly redundant calls, such as frogs, cicadas, crickets, mole crickets and conehead tettigoniids (e.g. Bennet-Clark 1970; Counter 1977; Stevens and Josephson 1977; MacNally and Young 1981; Prestwich and Walker 1981; Ryan et al. 1983; Kavanagh 1987; Forrest 1991). Metabolic measurements obtained from singing insects revealed a very inefficient way of sound production with only $0.05-2 \%$ (exceptional $6 \%$ for Requena verticalis) of energy invested in signalling mirrored in the acoustic power of the generated sound (Bailey et al. 1993; Prestwich 1994). This is mainly a consequence of a mismatch between the radiating structure and the emitted wavelength (Bennet-Clark 1971, 1975).

The low rate of conversion of metabolic energy into acoustic power is mainly determined by signal amplitude, duration, and rate (Prestwich 1994; Reinhold et al. 1998; McLister 2001; Robinson and Hall 2002). These signal parameters contribute additively to calling effort, which strongly correlates with calling energetics, both in insects and anurans (reviewed in Prestwich 1994; Gerhardt and Huber 2002). Since only males of good quality are supposed to uphold a high calling effort, costly acoustic advertisement signals are assumed an honest indicator of a signaller's quality (Zahavi 1975). 
Not surprisingly, choosy females prefer males in aggregations which call at a higher signal rate and duration (in frogs: Rand and Ryan 1982; Arak 1988; in ensiferan Orthoptera: Forrest 1983; Hedrick 1986; Bailey et al. 1990; Tauber et al. 2001). A correlation of signalling effort and male condition was confirmed in various diet studies in which males were set on different nutritional regimes (e.g. Gryllus lineaticeps: Wagner and Hoback 1999, for a field study of Gryllus campestris see Holzer et al. 2003). Compared to signalling in isolation, males respond to other males by increasing signalling effort (rate, duration) (e.g. Mygalopsis marki: Dadour 1989; Platycleis albopunctata: Latimer 1981; Cyphoderris buckelli: Morris et al. 2002).

However, in insects and anurans, there seems to exist a trade-off between chirp rate and chirp duration (Shaw 1968; Loftus-Hills 1974; Latimer 1981; Greenfield and Minckley 1993; Tejedo 1993; Greenfield 1994b). Anurans adjust call rate and call duration according to chorus size (Wells and Taigen 1986, 1989; Schwartz et al. 2002; Martínez-Rivera and Gerhardt 2008). For example, male Hyla versicolor decrease call rate and simultaneously increase call duration in a chorus, a behaviour that maintains the cost of calling at a rather constant level (Wells and Taigen 1986). Obviously, males in acoustic interactions uphold a certain duty cycle (proportion of signal ON-time) by adjusting signal rate and signal duration.

The mechanism allowing males to control duty cycle while signalling at various rates requires a high plasticity of signal production. In Pterophylla camellifolia signal duration is a function of the phase shift induced by an acoustic stimulus (Shaw 1968). Similarly, in the synchronizing snowy tree cricket Oecanthus fultoni leader males in an interaction increase chirp duration while follower males shorten their chirps (Walker 1969). A regulation of signal duration on a chirp-to-chirp basis may arise from limited energetic reserves associated with calling and allows males to maintain energetic costs in acoustic interactions at a rather constant level (Greenfield 1994a). These examples suggest that signallers suffer from a trade-off between signal attractiveness and calling energetics.

Calling in imperfect synchrony has usually a profound influence on a signaller's attractiveness. In Neoconocephalus spiza (Snedden and Greenfield 1998), Amblycorypha parvipennis (Galliart and Shaw 1996), Ephippiger ephippiger (Greenfield et al. 1997), Ligurotettix planum (Minckley and Greenfield 1995), Ligurotettix coquilletti (Greenfield et al. 1997) and Mecopoda elongata (Fertschai et al. 2007) females prefer males timing their signals in advance to others. This preference for leader males is less common in anurans, but upon existence, also has the potential to override preferences for other call parameters, like amplitude (Klump and Gerhardt 1992; Greenfield 1994b; Howard and Palmer 1995; Grafe 1996; Greenfield et al. 1997; Snedden and Greenfield 1998). Therefore, males consistently timing their chirps as a follower would suffer from a lower mating success. Thus, it is likely that males have evolved compensatory strategies.

Here, we investigated chirp plasticity in M. elongata and its consequence for signalling energetics. Acoustic monitoring and laservibrometry was used for the investigation of chirp production of solo singing males, after entrainment to a repetitive conspecific signal, as well as in male duets. We quantified the energetic costs of singing in a metabolic chamber, and studied the thoracic temperature of singing males with infrared thermography and a remotely operated infrared thermometer.

\section{Materials and methods}

Insects

The calling songs of $M$. elongata consist of chirps repeated in a highly regular manner with an average chirp period of $2.0 \mathrm{~s}$ (at an ambient temperature of $27^{\circ} \mathrm{C}$ ). The taxonomic relationship of species in the genus Mecopoda is uncertain, and there are several sibling species, which are morphologically similar with distinctly different song patterns. The insects in this study were originally collected in the field in Malaysia; their song is identical to the one described by Sismondo (1990) as "species S". The insects were reared at $27^{\circ} \mathrm{C}$ and $80 \%$ relative humidity on a $12: 12 \mathrm{~h}$ light:dark cycle. Soon after the onset of the dark phase, males start to sing for several hours. In acoustic interactions, males synchronise their chirps (Sismondo 1990). However, synchrony during acoustic interactions is not perfect and males time their chirps either as leader or as follower. A major determining factor for becoming either leader or follower for long periods of time is the solo chirp rate which allows faster solo chirping males to establish the leader role more frequently (Hartbauer et al. 2005; Hartbauer 2008).

Sound recordings and playback experiments

Recordings of solo song activity as well as singing during playback experiments were performed in a temperaturecontrolled incubator $\left(64 \times 55 \times 62 \mathrm{~cm} ; 27^{\circ} \mathrm{C}\right)$. The effect of echoes in the chamber was minimised by covering its inner surface with acoustic foam. The singing activity of males held in a $5 \times 5 \times 7 \mathrm{~cm}$ screen cage was recorded by means of tie-pin microphones positioned close to the insect. Due to a low sensitivity of this microphone, a mix of the recording with playback signals was strongly reduced. Only for the investigation of syllable amplitude of leader and follower signals, sound recordings were performed using a calibrated microphone (LD 2540, Type 4133, 
Larson Davis, Provo, UT, USA) attached to a sound level meter (CEL 414).

In order to entrain males to a repetitive pacer either a typical chirp of a solo singing male (281 ms in duration) or the combined signal of three males synchronizing their chirps in a small chorus (300 $\mathrm{ms}$ in duration) was used in playback experiments. Both conspecific signals were recorded using a calibrated microphone (see above). Exact timing of the playback signals was achieved by means of a custom-written sequencer script (Spike2, Cambridge Electronic Design, UK) controlling the DA output of a Power 1401 (Cambridge Electronic Design). A/D inputs of this device were used for simultaneously recording both the playback signal and the singing activity of males. To study the chirps of males timing their signals as leader and follower within a single song bout, males were entrained to a cyclic playback of the conspecific chirp by gradually decreasing the chirp period of the signal in steps of $3 \mathrm{~ms}$.

After attenuation and amplification (Heineken Inc., Germany) signals were broadcast to singing males through a dome tweeter (Dynaudio, Denmark). Unless stated otherwise, the SPL of signals used for entrainment was calibrated to $64 \mathrm{~dB}$ at the position of the singing male, using a sound level meter (for device description see above). This SPL corresponds to the amplitude of the loudest syllable and was peak equivalent to a $8 \mathrm{kHz}$ tone used for sound calibration. The timing of chirps with respect to stimulus onset as well as the duration of chirps was evaluated using a custom-written Spike2 script after setting a manual threshold for the detection of chirp onsets. Recorded chirps of the singing male were easily distinguished from the stimulus artefact by their amplitude.

\section{Recording of song interactions between two males}

Acoustic interactions of two males exhibiting a similar solo chirp period were studied in the same incubator as described above, at a constant temperature of $27^{\circ} \mathrm{C}$. Two males, each caged in a $5 \times 5 \times 7 \mathrm{~cm}$ box, were positioned along the diagonal axis of the incubator. An inter-male distance of about $5 \mathrm{~m}$ was mimicked by the sound attenuating property of acoustic foam (thickness $5 \mathrm{~cm}$ ) separating both males. This reduced chirp intensities to approximately $70 \mathrm{~dB}$ SPL. Tie-pin microphones mounted close to each male allowed us to record the acoustic interactions between pairs of males while reducing the cross-talk between both recording channels.

Recording of wing movements during stridulation

The movement of forewings during stridulation was monitored using a laser-Doppler-vibrometry device equipped with flexible fibre optics (OFV-3000 controller connected to a OFV-501 sensor head, Polytec Inc.) in a room with walls covered with acoustic foam (ambient temperature $24^{\circ} \mathrm{C}$ ). In order to improve the quality of the laser signal reflected from the left forewing a small piece of a self-attaching reflecting tape was mounted at the position of the stridulatory file. Since males usually did not move during singing, it was possible to focus the laser beam at this position for longer sequences. The velocity and displacement output of the laservibrometer was recorded together with the sound signal on a four channel Powerlab 4/25 (Model: ML845, AD Instruments, Germany). The sampling rate for all channels was set to $100 \mathrm{kHz}$. A condensor microphone (Larson Davis) attached to a sound level meter was used for sound recording.

\section{Calling metabolism}

Individual males were transferred into a metabolic chamber for a whole dark period in order to continuously measure $\mathrm{CO}_{2}$ production rate. The size of the metabolic chamber was adjusted to match the size of the bushcrickets, but still allowed males reduced movement. In order to control ambient temperature of the insect the metabolic chamber was placed into an incubator (Heraeus Inc.). The behaviour of the males inside the chamber was observed with an infrared video camera (Sony MiniDV Camcorder, DCRTRV14) connected to a video monitor. Males frequently moved around within the first $30 \mathrm{~min}$ after transfer to the metabolic chamber, whereas they rarely changed their positions later on. Thus, we are sure not to confuse metabolic rate for calling and locomotion.

The volumetric capacity of the metabolic chamber was $200 \mathrm{ml}$. A small electred microphone was integrated in the wall of the chamber to record male songs. Together with the $\mathrm{CO}_{2}$ production rate of the insect the air temperature of the metabolic chamber $\left(27 \pm 0.5^{\circ} \mathrm{C}\right)$ was measured by a thermocouple and was sampled in periods of $3 \mathrm{~s}$ by Centrol 4 software on a PC. Before starting a measurement, the metabolic chamber with the insect was submerged in water and a moderate overpressure was applied in order to check the sealing of the chamber. A differential URAS 14 gas analyser $(\mathrm{ABB})$ combined with a Brooks 5850S mass flow controller (0-1,000 ml/min; Brooks Instrument, Hatfield, USA) was used to continuously measure $\mathrm{CO}_{2}$ concentration in the metabolic chamber (Kovac et al. 2007). This was done by determining the difference in $\mathrm{CO}_{2}$ concentration of a constant flow of air $(150 \mathrm{ml} / \mathrm{min})$ before and after passing the metabolic chamber. The air was taken from outside the laboratory. Before entering the measurement system it had to pass a 101 canister and a 51 bottle to smooth any variations in outside $\mathrm{CO}_{2}$ concentration. Before the air entered the reference and measurement tubes of the URAS (heated to $60^{\circ} \mathrm{C}$ ) it was dried by two 
Peltier-driven cold traps $\left(10^{\circ} \mathrm{C} ; \mathrm{ABB}\right) . \mathrm{CO}_{2}$ production rate of the insects was obtained by calculating the product of the measured $\mathrm{CO}_{2}$ concentration with the actual air flow. Calibration of the system was automatically performed every $2 \mathrm{~h}$ both in zero point (against air from outside of the laboratory) and end point (with internal calibration cuvettes) after switching the air flow to bypass the measurement chamber. The calibration of the $\mathrm{CO}_{2}$ measuring system was checked by calibrated $\mathrm{CO}_{2}$ injections into the metabolic chamber. The resolution of the $\mathrm{CO}_{2}$ measurement equipment was $<0.2 \mathrm{ppm}$. Measurement accuracy was $<2 \mathrm{ppm}$. The volumes of $\mathrm{CO}_{2}$ production reported in this paper refer to standard (STPS) conditions $\left(0^{\circ} \mathrm{C}\right.$, $101.32 \mathrm{kPa}=760$ Torr). Because of the tube length between the metabolic chamber and the $\mathrm{CO}_{2}$ measuring device a delay of $27.5 \mathrm{~s}$ was measured at an air flow of $150 \mathrm{ml} / \mathrm{min}$. Therefore, all measurements have been compensated for this delay.

The mass specific metabolic rate of a singing male was calculated by dividing the average $\mathrm{CO}_{2}$ production rate in a steady-state section of the song bout (average duration of $180 \mathrm{~s}$ ) by its fresh weight. The conversion of metabolic turnover $\left(\mathrm{CO}_{2}\right.$ production) to metabolic power was accomplished by assuming a respiratory quotient (RQ) of $\sim 0.85$ of singing animals (e.g. Prestwich and O'Sullivan 2005; compare also Harrison 1989) to estimate $\mathrm{O}_{2}$ consumption, and a caloric equivalent of oxygen of $\sim 20.288 \mathrm{~kJ} / 1 \mathrm{O}_{2}$ by assuming combustion of $44 \%$ carbohydrates, $44 \%$ fat and $12 \%$ proteins. By this means, massspecific metabolic power (in $\mathrm{mW} \mathrm{g}^{-1}$ ) could be estimated by multiplying metabolic turnover $\left(\mu \mathrm{l} \mathrm{CO}_{2} \min ^{-1} \mathrm{~g}^{-1}\right.$ ) with a factor of 0.3978. Mass-specific energetic costs per chirp (in $\mathrm{J}_{\text {chirp }}^{-1} \mathrm{~g}^{-1}$ ) were estimated by multiplying mean $\mathrm{CO}_{2}$ production $\left(\mu \mathrm{l} \operatorname{chirp}^{-1} \mathrm{~g}^{-1}\right.$ ) with a factor of 0.020288 .

We performed metabolic measurements with males inside the chamber while they were entrained to a regularly repeated solo chirp. However, due to the small and closed volume of the chamber the SPL of the playback chirp could not be determined in the same way as under normal, far field conditions. Therefore, the SPL was adjusted to a level where all 12 males examined showed reliable phase-locked singing.

\section{Infrared thermography}

The thoracic surface temperature of singing and resting males was either recorded with a remote-operating infrared thermometer (Raytec Phototemp MX6, accuracy: $0.75^{\circ} \mathrm{C}$ at $25^{\circ} \mathrm{C}$ ) equipped with a laser indicating the measurement area, or with a SC2000-NTS infrared camera (FLIR; Kovac et al. 2010; Stabentheiner et al. 2010) calibrated in regular intervals against a custom-made Peltier-driven reference radiator (accuracy $0.7^{\circ} \mathrm{C}$ ). Insect cuticle infrared emissivity was assumed to be 0.97 as in other insects (Stabentheiner and Schmaranzer 1987; Kovac and Stabentheiner 1999). Measurements were taken from three different species: On the one hand, males of $M$. elongata with the above described chirp period of $2 \mathrm{~s}$. In addition, we used another Mecopoda species from South India (Bangalore) with a chirp period of $0.5 \mathrm{~s}$, and a further species with a continuous trill collected in Khao Luang (Thailand). All infrared thermometer measurements were performed at an ambient room temperature of $26^{\circ} \mathrm{C}$ and a relative humidity of $75 \%$.

\section{Statistics}

We performed all statistical calculations in Sigmaplot 11.0 (Systat Software Inc.). Differences between two groups were tested for significance by application of a $t$ test. Multigroup comparison was performed by application of an ANOVA. Data was checked for linear distribution using a Shapiro-Wilkinson test. A correlation between parameter pairs was tested for significance by means of a Spearman rank order correlation and by linear regression (when data were normally distributed).

\section{Results}

Chirp production of solo singing males

Mecopoda elongata males singing in isolation produced songs that consisted of regularly repeated chirps with an average duration of $273 \mathrm{~ms}$ (minimum $=250 \mathrm{~ms}$, maximum $=350 \mathrm{~ms}, N=12$ ). The mean chirp period of solo singing males was $2.02 \pm 0.08 \mathrm{~s}$ (mean of 32 males; average ambient temperature $26.0-27^{\circ} \mathrm{C}$; see also Hartbauer et al. 2006). Chirps consisted of 13-20 syllables successively increasing in amplitude. Each syllable could be subdivided into a loud hemi-syllable followed by a soft one (Fig. 1a). The average syllable repetition rate was in the range of $53-60 \mathrm{~Hz}$, corresponding to a syllable period of 16-18 ms. Although males singing in isolation exhibited a rather steady chirp period, the duty cycle (proportion of signal ON-time) typically showed a gradual decrease over the time of a singing bout (see example in Fig. 1b). This was the consequence of a gradual decrease in chirp duration, rather than chirp rate, which remained almost constant.

Ensiferan insects generate sound by frictional forces by rubbing both front wings (tegmina) against each other, an activity called stridulation. Laservibrometry of tegmen movement revealed that each hemi-syllable is generated during different wing cycles. Loud hemi-syllables were generated in the course of wing opening; in contrast, wing closing resulted in the production of soft hemi-syllables 


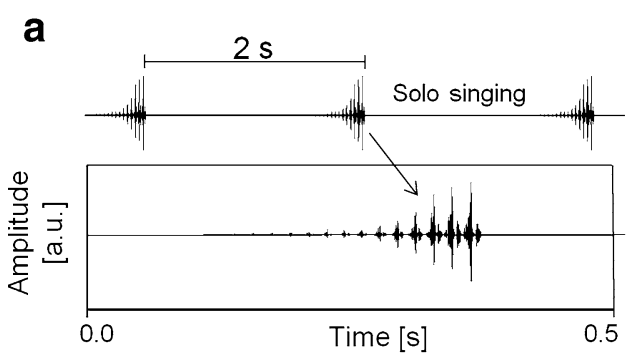

b

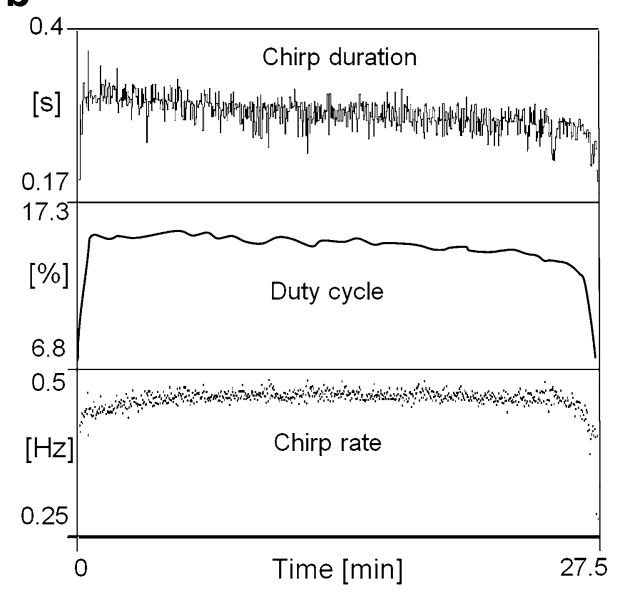

Fig. 1 Sound production in M. elongata and chirp plasticity. a Chirps of solo singing $M$. elongata male. b Chirp duration and rate, and duty cycle (proportion of signal ON-time) in a typical song bout of a solo singing male. $\mathbf{c}$ Laservibrometry of forewing movements during chirp production shown as displacement and velocity. Wing oscillations are apparent in the spiky velocity trace (upper trace). Note that loud

(see Fig. 1c). The gross movement pattern of front wings was only partially mirrored in the gradual increase of syllable amplitude (Fig. 1d). Soft syllables produced right after chirp onsets were generated by a similar wing displacement compared to syllables of medium amplitude. The average maximum speed of tegmen movement was higher during opening compared to wing closing ( 35 vs. $20 \mathrm{~cm} / \mathrm{s} ; 4$ males, 33 chirps, ambient temperature $24^{\circ} \mathrm{C}$ ). The file and scraper mechanism sets wings into vibration, which was mirrored in the oscillations of the velocity output of the laservibrometer (Fig. 1c, upper trace).
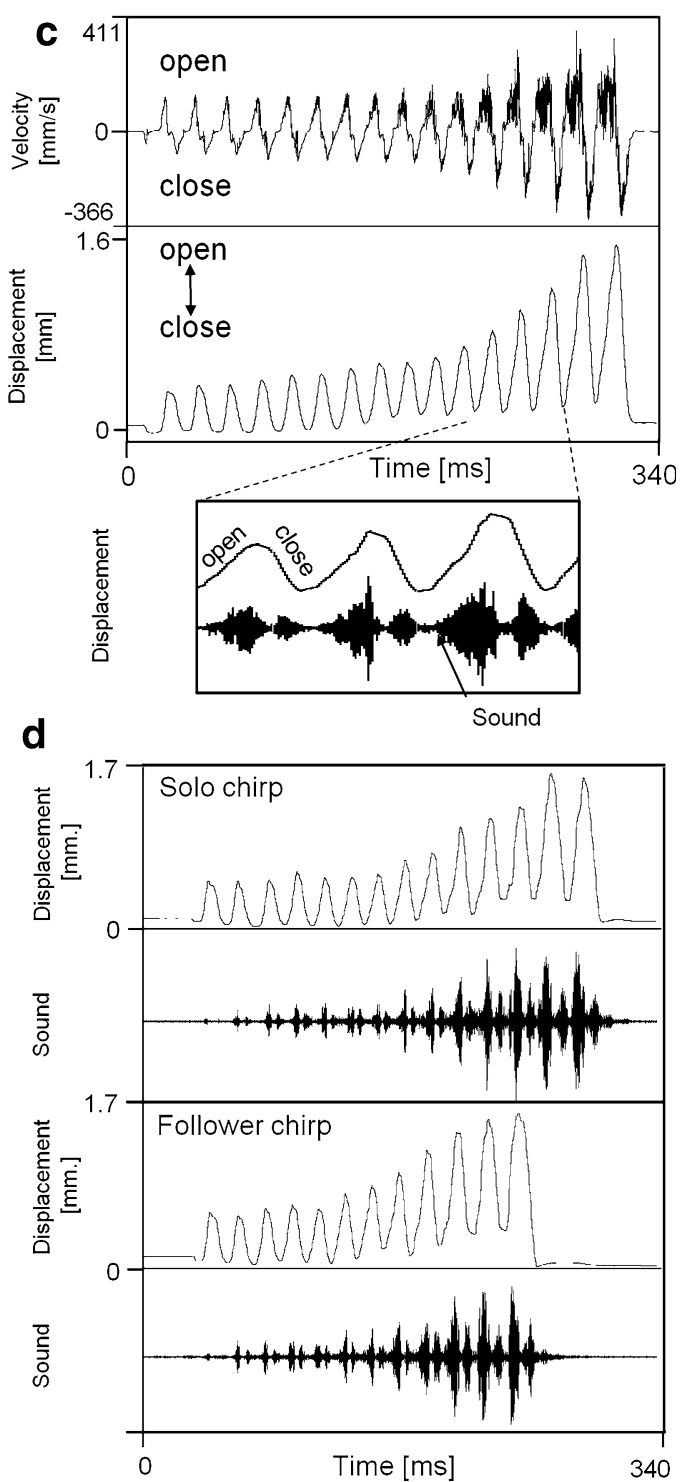

hemi-syllables are generated during wing opening and soft hemisyllables during wing closing movements (see magnification in the bottom trace). d Comparison of a chirp produced by a male during solo singing, and as follower chirp in an entrainment experiment. Note the reduction in chirp duration of the follower chirp. For average values see Table 1

\section{Entrained singing}

In experiments in which males were entrained to a periodic conspecific signal (pacer) broadcast at a slightly higher signal rate compared to a male's solo chirp rate, males usually timed their chirps after the onset of the pacer. Such follower chirps were shorter compared to solo chirps or chirps timed in advance to the entraining stimulus (leader chirps) (Fig. 1d; lower traces). Follower chirps consisted of fewer soft syllables and exhibited a slightly higher syllable rate compared to solo singing ( 57 vs. $53 \mathrm{~Hz}$ at an ambient 
temperature of $24^{\circ} \mathrm{C}$ ). The power spectral density of conspecific signals showed two maxima, one in the frequency range of about $7 \mathrm{kHz}$ and another one at roughly $50 \mathrm{kHz}$. Leading and follower chirps were not different in their spectral composition (data not shown).

When the entraining signal gradually increased in rate, males increased their chirp rate, thus maintaining a 1:1 phase-locked synchrony. As a consequence, the time difference between signal onset and chirp onset $(\Delta t)$ gradually increased at higher signal rates (Fig. 2a, top trace). Nevertheless, the duty cycle (signal ON-time) remained at a rather constant level. This was the consequence of shorter chirps generated at higher chirp rates. During synchronous entrainment, chirp duration was reduced as $\Delta t$ increased (black dots in Fig. 2b). However, a further decrease of the signal period below $1.39 \mathrm{~s}$ abolished 1:1 synchrony (indicated by a dashed vertical line in Fig. 2a and open symbols in Fig. 2b). In this situation, males timed their signals at various $\Delta t$; sometimes males extended the duration of chirps significantly after timing their signals right at the end of the entraining signal. Table 1 summarizes the plasticity of chirps generated in entrainment experiments with a gradually increasing pacer rate.

The average duration of leader chirps during synchronous entrainment to a conspecific signal at a constant signal period of $2.0 \mathrm{~s}$ was significantly longer compared to follower chirps (Fig. 2d, $P<0.001$, paired $t$ test, 11 males). Moreover, the same males singing in isolation produced significantly longer chirps (solo chirps) compared to the entrainment situation in which they generated signals as follower (273.7 vs. $212.2 \mathrm{~ms}$, average of 11 males; Fig. 2d). In contrast to follower signals, solo chirps were

b
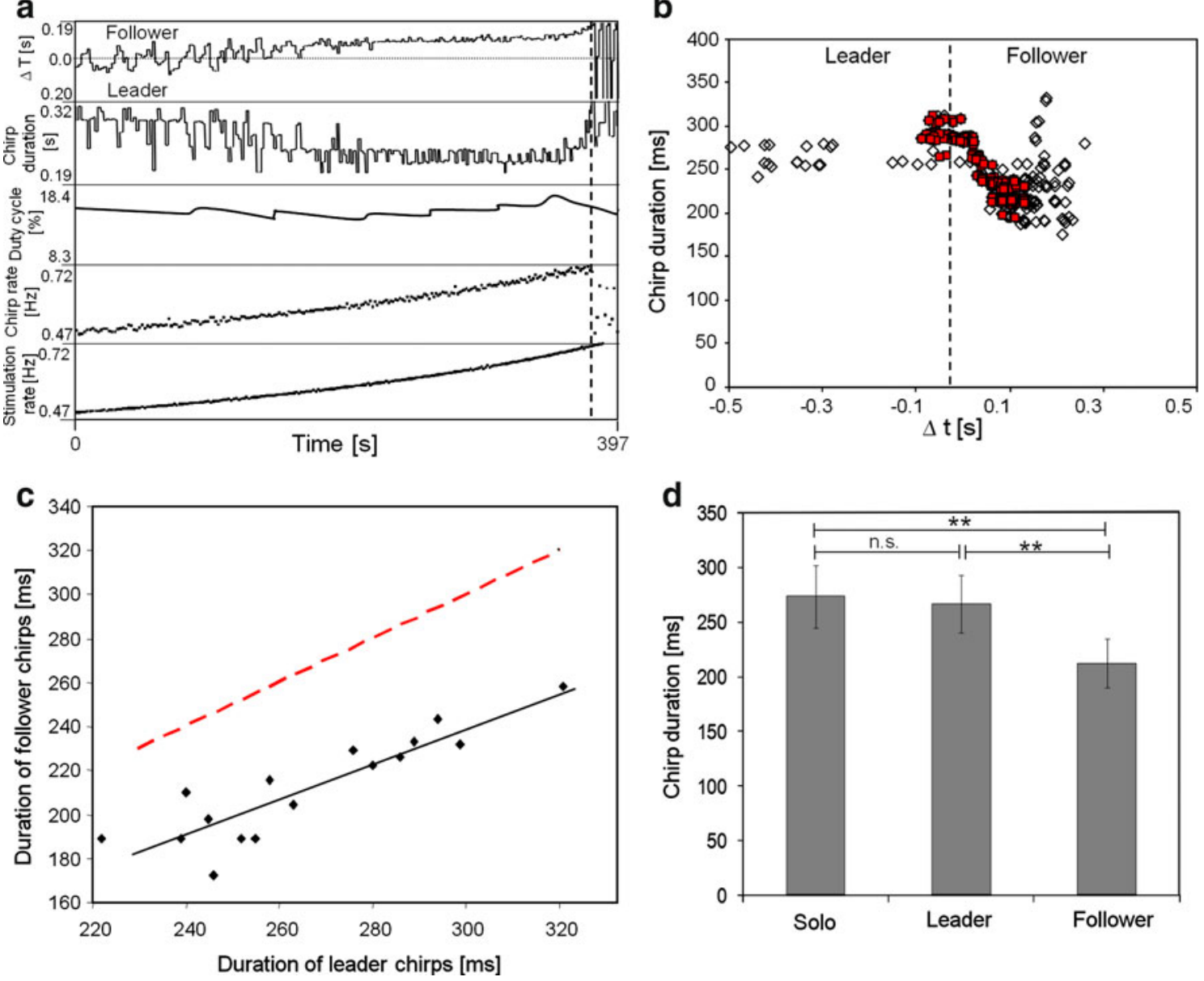

Fig. 2 Signal plasticity in entrainment experiments. a Duty cycle, chirp duration, chirp rate and time difference between signal onset and chirp onset $(\Delta t)$ of a male entrained to a periodic conspecific signal gradually increasing in rate (bottom trace). Stable 1:1 phaselocked entrainment occurred up to signal periods of $1.39 \mathrm{~s}$ (vertical dotted line). b Chirp duration during entrainment (same as in a) as a function of $\Delta \mathrm{t}$. Red squares duration of chirps produced during stable 1:1 synchrony, open symbols chirps generated after synchrony was lost. c Relationship between the average duration of leader chirps during synchronous entrainment and average duration of follower chirps (pacer period $2.0 \mathrm{~s} ; 16$ song bouts, 11 males). Slope of the regression line is 0.79 and correlation coefficient 0.606 . Dashed red line indicates the absence of chirp plasticity. d Average duration of solo chirps, leader and follower chirps during synchronous entrainment (pacer period $2.0 \mathrm{~s}$ ). Follower chirps were significantly shorter than leader or solo chirps $(P<0.001$, paired $t$ test, $N=11$ males $)$ 
not different from leader chirps with respect to chirp duration. On average, males shortened their follower chirps by $33 \%$, and males generating longer chirps as leader shortened follower chirps slightly further (see divergence in slope between the solid and broken line in Fig. 2c).

\section{Duetting males}

In male duets, one male typically leads the other when his solo chirp period is shorter than that of his opponent. However, in rare cases both males exhibited a similar average solo chirp period causing leader and follower roles to switch back and forth between males ( $\Delta t$ in Fig. 3a). Such duets were characterised by only little variability in chirp rate and duty cycle, but strong fluctuations in chirp duration of both males (Fig. 3a). Generally, leader chirps were longer compared to follower chirps, which showed a gradual decrease of chirp duration with increasing $\Delta t$ (Fig. 3b). Chirp shortening contributed to a temporal overlap of the end of leading chirps with loud syllables of follower chirps. This was obvious by plotting $\Delta t$ of chirp onsets against the time difference of chirp endings (Fig. 3c). A deviation of the fitted curve from the dotted line indicates signal plasticity. Notably, shortening follower chirps was not correlated with gaining the leader role in the subsequent cycle; rather, followers achieved a stronger overlap with leading chirps, depending on the amount of chirp shortening and leader chirp duration. Furthermore, the signal plasticity did not result in a synchronisation of syllables (data not shown).

\section{Influence of pacer amplitude and duration}

Chirp plasticity may also be influenced by the amplitude of the entraining stimulus. Therefore, males were entrained to a periodic conspecific signal (solo chirp) presented at three different amplitudes (50, 60 and $70 \mathrm{~dB}$ SPL) gradually increasing signal rate. Results show that follower chirps were more strongly reduced in duration at higher stimulus levels $(60$ and $70 \mathrm{~dB})$. At the same time, a higher SPL increased the range of $\Delta t$ in which males shortened their follower chirps.

In addition, we investigated the plasticity of follower chirps in entrainment experiments with 4 stimuli: solo and chorus chirp and signals consisting of either two or four syllables, all presented at the same peak amplitude $\left(64 \mathrm{~dB}_{\mathrm{pe}}\right.$ SPL). Males shortened their follower chirps during synchronous entrainment with either a solo chirp or a chorus signal in a similar fashion (Fig. 4a). However, the range of $\Delta t$ in which males shortened their follower chirps was significantly smaller during entrainment to a pacer consisting of only two or four syllables. Interestingly, at signal periods shorter than $1.62 \pm 0.14 \mathrm{~s}$ (mean of 11 males, $27^{\circ} \mathrm{C}$ ) males
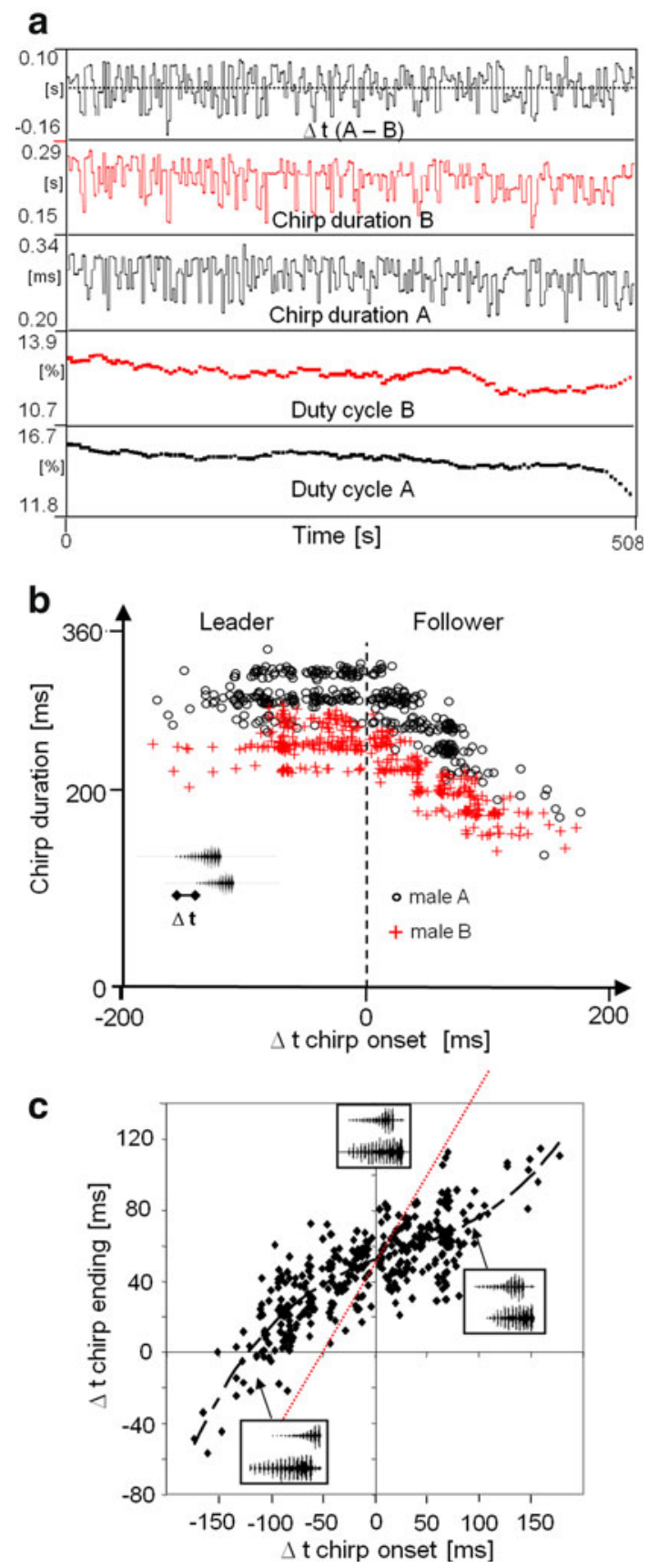

Fig. 3 Signal plasticity in a male duet. a Chirp duration, duty cycle and time difference between leader and follower chirp onset $(\Delta t)$ during a 8 min sequence of a 1:1 phase locked duet of two males with similar solo chirp periods $(2.2 \mathrm{~s})$. b Chirp duration of both males shown as a function of $\Delta t$. Note that chirps of male A and B differed in duration, but both males produced shorter chirps when singing as followers. c Relationship between the time difference of chirp onsets and chirp endings. The dotted red line indicates a situation without any chirp plasticity

lost 1:1 synchrony with the entraining signal and it frequently happened that follower chirps were even longer compared to leader chirps (arrows in Fig. 4a). This was 
a

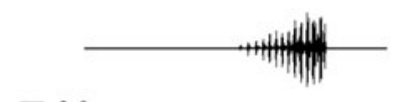

\#1

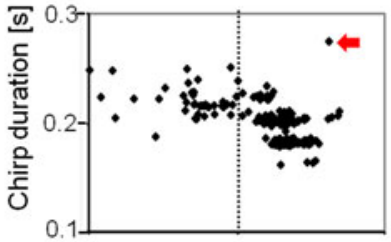

\#2
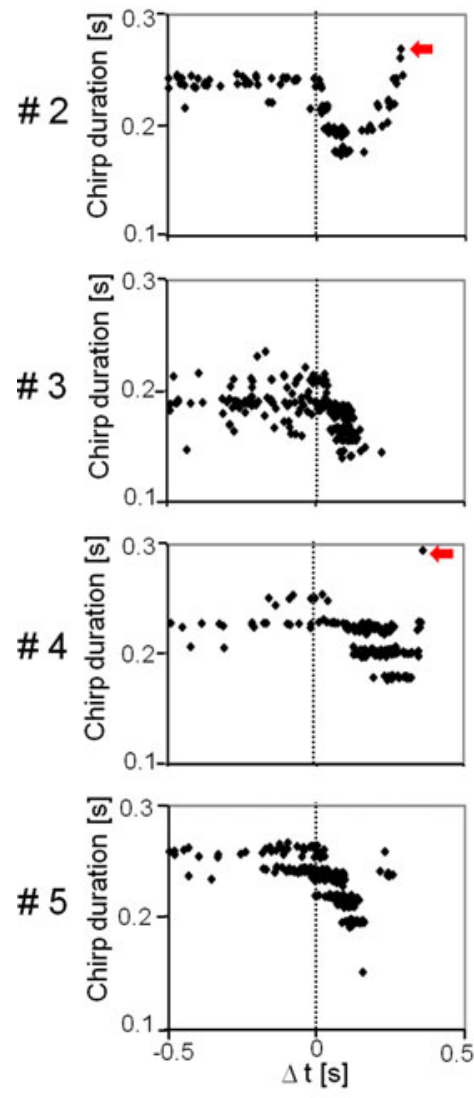

b

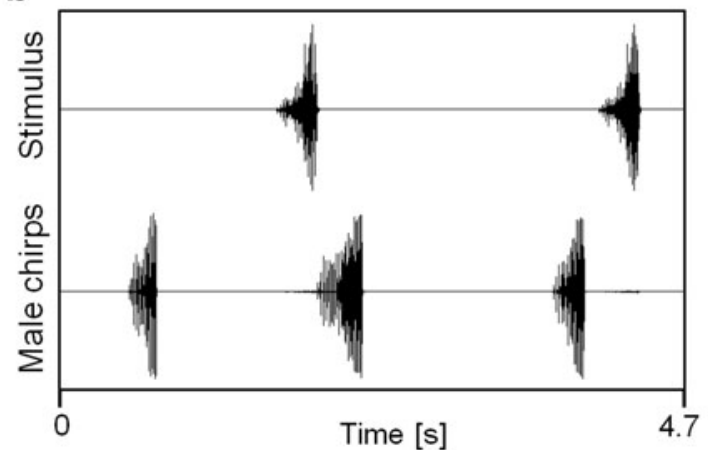

Fig. 4 Signal plasticity during entrainment to conspecific and artificial signals. Response of five males entrained to different pacers gradually increasing in rate: a solo chirp, a chorus signal and artificial signals consisting of either 2 or 4 "Mecopoda syllables". The duration of chirps is plotted as a function of the time difference
2 syllables

4 syllables
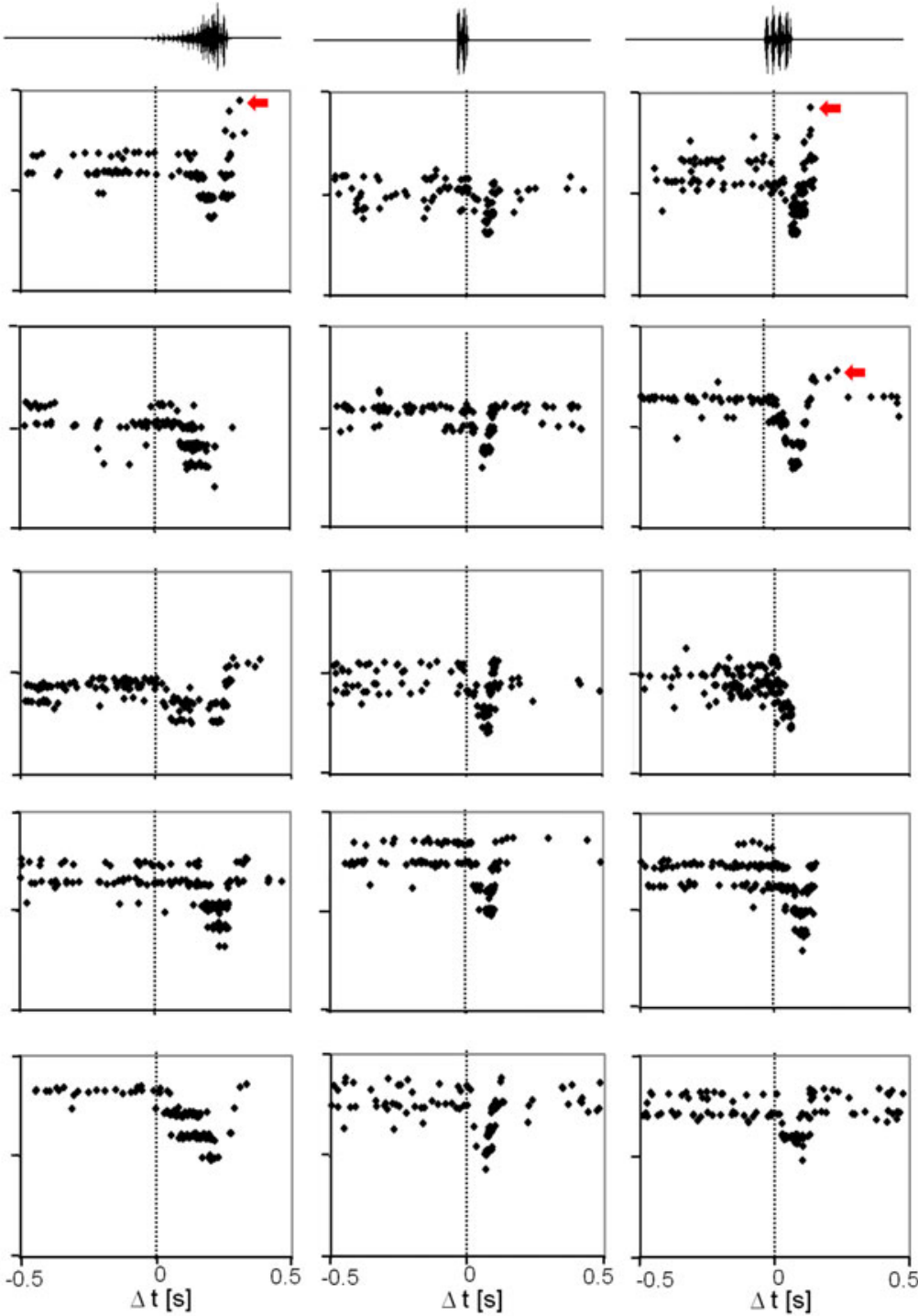

C

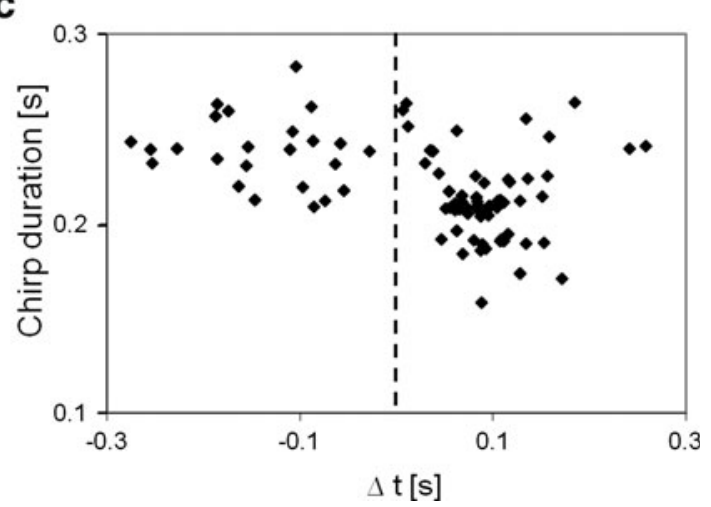

between pacer onset and chirp onset $(\Delta t)$. Red arrows indicate extraordinary long chirps following the end of the entraining signal after synchrony was lost (see one example in b). c Chirp duration of six males entrained to a repetitive conspecific signal randomly timed within a signal period of $2.0 \pm 0.3 \mathrm{~s}$ as a function of $\Delta t$ 

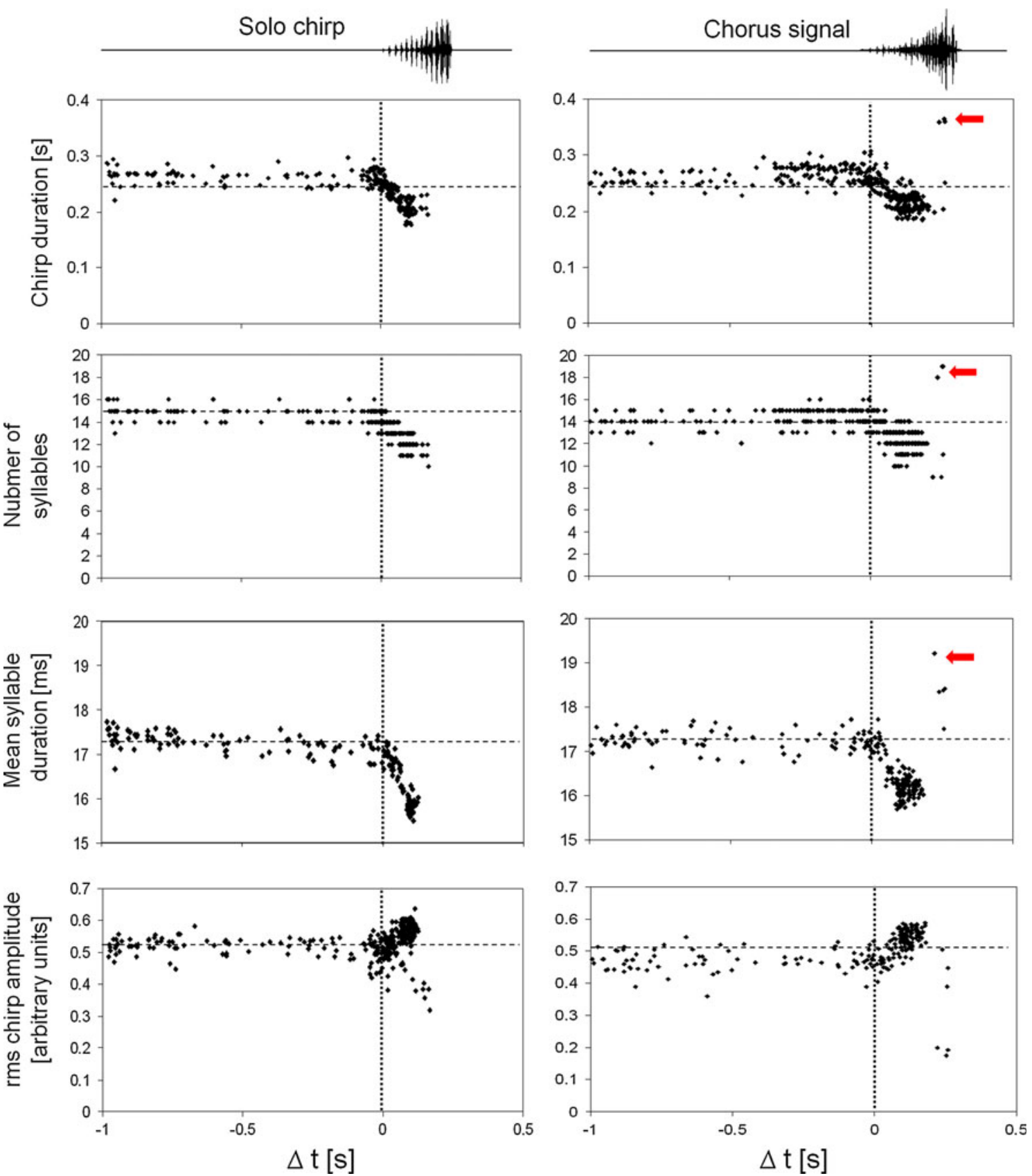

Fig. 5 Detailed analysis of chirp plasticity. Response of one male entrained either to a periodic solo chirp or a chorus signal gradually increased in signal rate (SPL of $70 \mathrm{~dB}$ ). Red arrow indicates extraordinary long chirps, high number of syllables and syllable duration

always the case when males timed their chirps right at the end of the entraining signal (see example in Fig. 4b).

In addition, the plasticity of follower chirps was investigated in entrainment experiments in which the onset of the entraining signal could not be anticipated, but still was presented in a periodic fashion. This was achieved by entraining males to a conspecific signal timed randomly in a time period of 2,000 $\pm 300 \mathrm{~ms}$. In this experiment, the extent of chirp shortening was similar compared to entrainment experiments in which a conspecific signal was repeated at a constant rate of $2.0 \mathrm{~s}(46 \mathrm{vs} .54 \mathrm{~ms}$, average of 6 males; Fig. 4c).

A detailed analysis of chirps generated at various $\Delta t$ revealed that males not just reduced the number of syllables, but also decreased the duration of syllables when signalling as follower (see example in Fig. 5). This chirp plasticity was observed in entrainment experiments using as pacer either a solo chirp or a synchronized chirp of three males. The example demonstrates that the shortening of the chirps as followers is due both to a reduction of syllable 

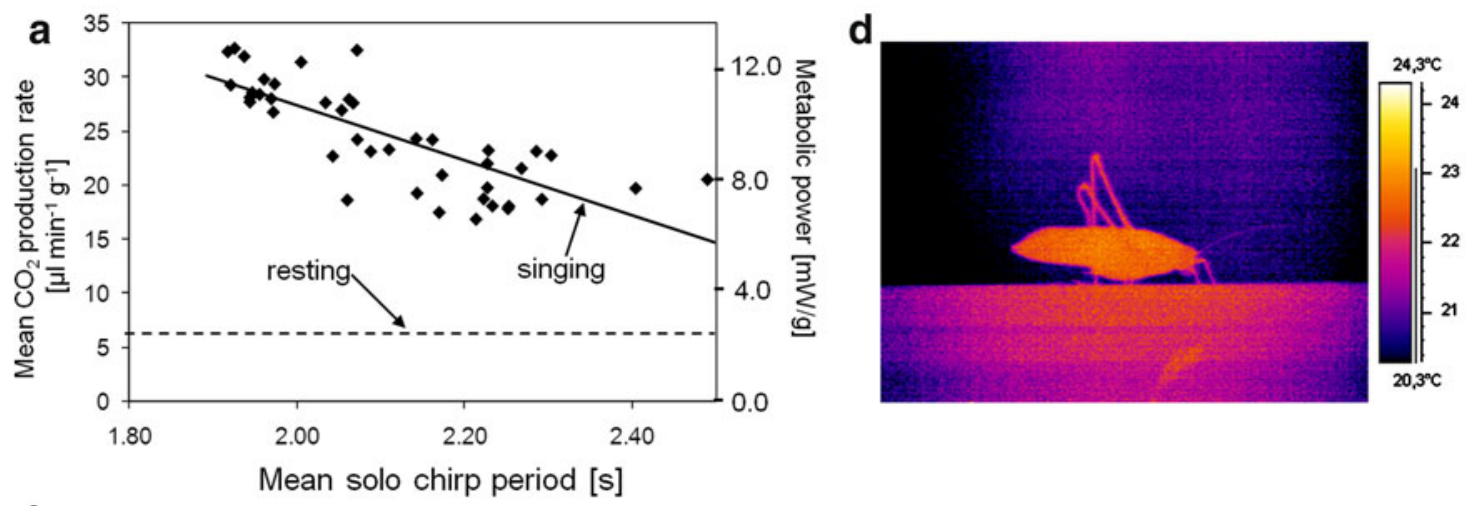

b
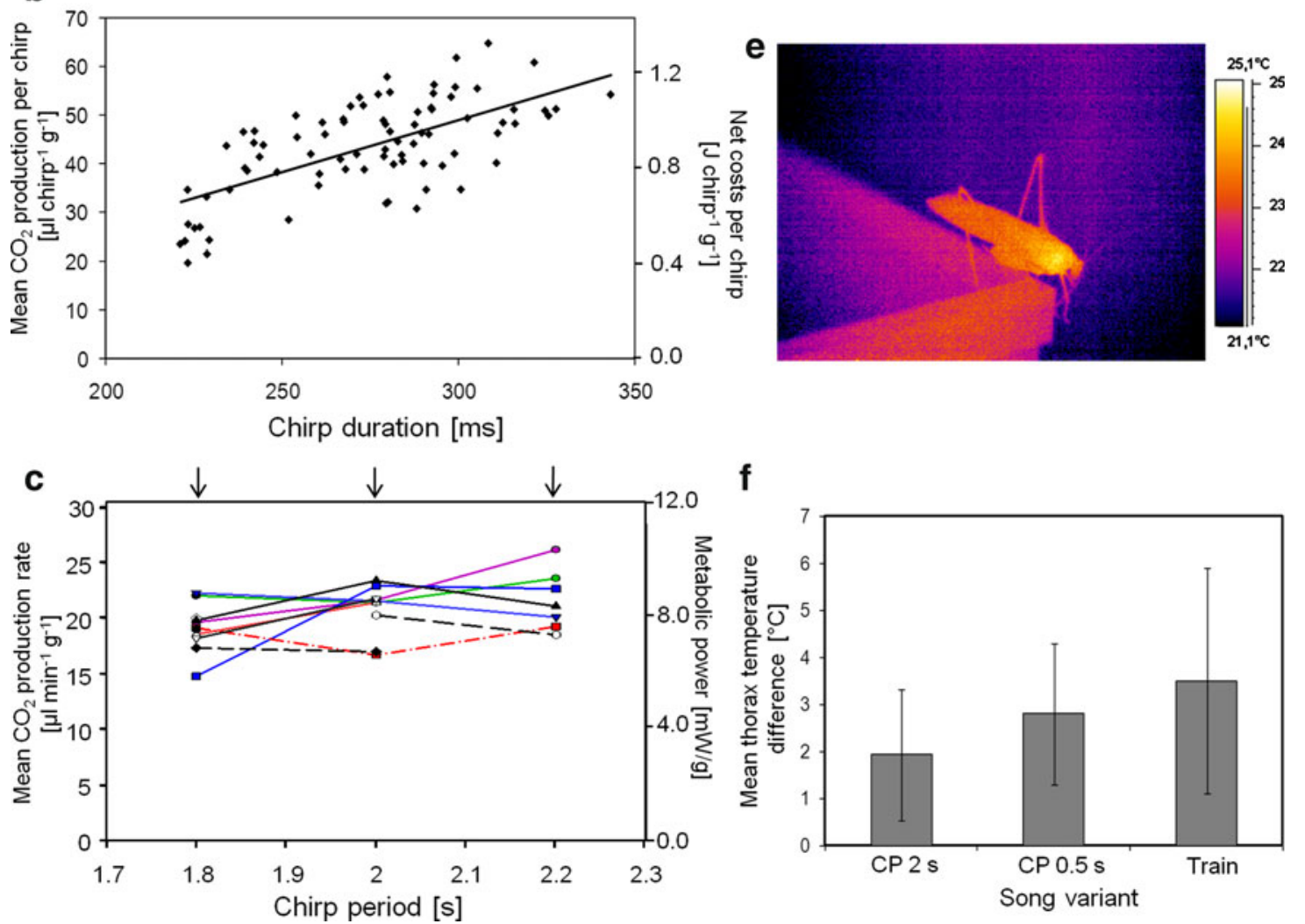

Fig. 6 Calling metabolism and body temperature of singing males. a Relationship of the average mass-specific $\mathrm{CO}_{2}$ production rate of solo singing males and their chirp period $\left(R^{2}=0.65, \mathrm{CO}_{2}\right.$ rate $=$ $(-25.524 \times$ solo $\mathrm{CP})+78.414, N=12$ males, about 3 songs per male). b Average mass-specific volume of $\mathrm{CO}_{2}$ produced per chirp as a function of chirp duration $\left(R^{2}=0.42, \mathrm{CO}_{2}\right.$ volume $=(0.2151 \times$ chirp duration) $-15.386, N=11$ males). c Average mass-specific

number and duration (by as much as $2 \mathrm{~ms}$ ). Interestingly, the root-mean-square amplitude of follower chirps was slightly higher compared to leading chirps, because shorter chirps have fewer syllables of low and medium amplitude. The increase in amplitude of successive syllables in follower chirps was significantly faster compared to leader chirps $(P<0.05$, paired $t$ test, number of chirps: 66-389, 3 males).
$\mathrm{CO}_{2}$ production rate of 12 males entrained to three different signal periods indicated by arrows. Data in $\mathbf{b}$ and $\mathbf{c}$ are from the same experiments. Infrared thermogram of a resting (d) and singing (e) male. f Average difference in surface thorax temperature between singing and resting males in three species of the genus Mecopoda (measured by means of an infrared thermometer)

Signalling metabolism and body temperature

The solo singing metabolic rate of $24.6 \pm 4.8 \mu 1 \mathrm{~min}^{-1} \mathrm{~g}^{-1}$ was considerably higher than the resting metabolic rate of $6.33 \pm 0.84 \mu \mathrm{min}^{-1} \mathrm{~g}^{-1}$ (10 males; ambient temperature $27^{\circ} \mathrm{C}$ ). The resting metabolic rate increased with male weight: $V \mathrm{CO}_{2}\left(\mu \mathrm{min}^{-1}\right)=8.7452 \times$ weight -4.5561 $\left(R^{2}=0.7908\right)$. Respiratory measurements revealed that 
Table 1 Signal plasticity of ten males entrained to a periodic conspecific signal gradually increasing in rate (stimulus amplitude $=70 \mathrm{~dB}$ SPL)

\begin{tabular}{llllll}
\hline Male \# & $\begin{array}{l}\text { Chirp } \\
\text { duration, } \\
\text { L (ms) }\end{array}$ & $\begin{array}{l}\text { Chirp } \\
\text { duration, } \\
\text { F (ms) }\end{array}$ & $\begin{array}{l}\text { Chirp } \\
\text { shortening } \\
(\mathrm{ms})\end{array}$ & $\begin{array}{l}\Delta t \text { leading } \\
\text { to chirp } \\
\text { shortening }(\mathrm{ms})\end{array}$ & $\begin{array}{l}\text { L-F syllable } \\
\text { difference }(N)\end{array}$ \\
\hline 1 & 217.8 & 193.0 & 24.8 & $140-290$ & 1.2 \\
2 & 238.4 & 208.3 & 30.1 & $70-316$ & 1.5 \\
3 & 202.3 & 168.4 & 33.9 & $51-122$ & 1.5 \\
4 & 203.9 & 185.0 & 18.9 & $140-268$ & 1.1 \\
5 & 246.3 & 224.3 & 22.0 & $100-274$ & 0.8 \\
6 & 262.7 & 221.0 & 41.7 & $60-252$ & 1.7 \\
7 & 249.8 & 216.3 & 33.5 & $93-287$ & 1.6 \\
8 & 236.9 & 221.1 & 15.8 & $61-246$ & $30-127$ \\
9 & 278.6 & 212.0 & 66.6 & $52-173$ & 3.2 \\
10 & 278.2 & 210.1 & 68.1 & $81.7-235.5$ & 3.9 \\
Mean & 241.5 & $206.0^{*}$ & 35.5 & $35.2-69.6$ & 0.8 \\
\hline SD & 27.5 & 18.2 & 18.4 &
\end{tabular}

$L$ leader, $F$ follower, $\Delta$ t delay between leader and follower signal onset

* Significant difference $(P<0.001)$ between leader and follower signals (paired $t$ test)

males singing at higher chirp rates during solo singing suffered from higher mass-specific metabolic costs (Fig. 6a, $N=12, R^{2}=0.65 ; P<0.001$, Spearman rank order correlation). In experiments in which males were entrained to a repetitive chirp presented at three different signal periods (1.8, 2.0 and $2.2 \mathrm{~s}$ ) the mass-specific metabolic rate of singing males showed a significant positive correlation with the duty cycle $\left(R^{2}=0.33 ; P<0.001\right.$, Spearman rank order correlation, $N=12$ males). Furthermore, a significant positive correlation between chirp duration and the average amount of $\mathrm{CO}_{2}$ produced per chirp revealed that longer chirps are energetically more expensive to produce compared to shorter ones $\left(R^{2}=0.42 ; P<0.001\right.$, Spearman rank order correlation, $N=11$ males, Fig. 6b). However, during synchronous entrainment to three different signal rates of a conspecific pacer the average $\mathrm{CO}_{2}$ production rate remained rather constant $\left(R^{2}=0.17 ; P=0.12\right.$, Two-way ANOVA with male identity as a co-variable, $N=12$ males; Fig. $6 \mathrm{c}$ ). This result is the consequence of a gradual reduction of chirp duration with increasing rate of the pacer $\left(R^{2}=0.60 ; P<0.001\right.$, Two-way ANOVA with male identity as a co-variable, $N=12$ males).

Infrared thermography revealed a slight increase of thorax temperatures of singing males (Fig. 6e) compared to resting males (Fig. 6d). The surface temperature of the thorax of singing males belonging to three different song variants of the genus Mecopoda (10 individuals per group) increased with calling effort (Fig. 6f). A temperature difference between singing and resting males of only $2^{\circ} \mathrm{C}$ was found in the chirping species with an average chirp period of $2.0 \mathrm{~s}$ ("species $\mathrm{S}$ "), whereas an increase of about $3^{\circ} \mathrm{C}$ was found in a chirping song variant with a chirp period of $0.5 \mathrm{~s}$. In a trilling species generating a train of syllables, the difference amounted to $3.65^{\circ} \mathrm{C}$.

\section{Discussion}

Females in chorusing insects and anurans often prefer the leader of two conspecific signals broadcast within a brief time interval (Whitney and Krebs 1975; Dyson and Passmore 1988; Klump and Gerhardt 1992; Howard and Palmer 1995; Grafe 1996; Snedden and Greenfield 1998; Greenfield and Rand 2000; Bosch and Márquez 2002). As a consequence, males should compete for the leader role in acoustic interactions and avoid the generation of less attractive follower signals. This is true for Neoconocephalus spiza where the leader and follower roles often switch between males (Snedden and Greenfield 1998). In Mecopoda elongata, however, males often time their signals as followers for long periods of time, both in a male duet and during entrainment experiments. How can the follower strategy survive in a population over evolutionary time, if it is not favoured by sexual selection through female choice? In the present paper, we addressed two possible solutions to this question, one considering signal plasticity in these interactions, the other higher costs associated with the leader strategy compared to the follower.

\section{Signal plasticity in M. elongata}

The mechanism responsible for controlling chirp duration is positively influenced by the duration and amplitude of the pacer (Fig. 4a). This favours signal jamming during acoustic interactions of neighbouring males. Even when males were unable to anticipate the exact timing of the next acoustic signal, they were able to respond to the concurrent signal by reducing chirp duration within a very short time lag. This mechanism responsible for signal plasticity may be similar to the mechanism leading to a synchronous 
display of advertisement signals in Oecanthus fultoni (Walker 1969; Greenfield 1994a), North American fireflies (Photinus sp.) and the frog Smilisca sila (Ryan 1986). In $O$. fultoni follower signals are also shorter, but different to $M$. elongata, males also lengthen their signals timed in advance to competitors. However, synchrony in both species is achieved in a different way, most obvious in a comparison of the phase response characteristic of the song oscillator (Hartbauer et al. 2005). In acoustic interactions with competitors tree cricket males reduce the chirp duration in the disturbed cycle and shorten the cycle length in the subsequent cycle. This mechanism improves the likelihood of becoming leader in the subsequent cycle and is responsible for trading leader roles back and forth. This is different to M. elongata where followers shorten their chirp as well as their chirp period in the disturbed cycle. Therefore, duetting males rarely trade leader and follower roles unless solo chirp periods are similar.

$O$. fultoni signal at a rate five times higher compared to M. elongata. This difference suggests chirp plasticity in $O$. fultoni to be the outcome of energetic constraints associated with the production of costly advertisement signals (Greenfield 1994a). Mecopoda males exhibit a metabolic rate during solo singing which is four times higher compared to resting (see also Hartbauer et al. 2006), comparable to the metabolic rate of chirping in Teleogryllus commodus (Kavanagh 1987). This renders singing in $M$. elongata energetically less demanding compared to anuran vocalisation ( $\sim 12$ times resting metabolic rate) or the energy expenditure found in trilling crickets $(\sim 9$ times resting metabolic rate; Prestwich 1994, Gerhardt and Huber 2002). A rather moderate energy consumption associated with chirping at a period of about $2 \mathrm{~s}$ may be responsible for the result of a diet study in which solo chirp rates of $M$. elongata males were not different between two male groups either set on a high or low nutrition diet (Hartbauer et al. 2006). This is surprising considering the fact that faster solo singing males suffered from higher energetic costs (Fig. 6a) and strongly suggests that even low-quality males are able to uphold a high chirp rate.

Despite a rather moderate energy expenditure associated with singing in M. elongata, it is surprising that the observed signal plasticity is associated with energy saving while signalling at higher rates. This suggests the shortening of follower signals to be a by-product of the mechanism with which males establish synchrony in a chorus situation, but less likely to be the outcome of energetic limitations associated with acoustic signal production. This view gets support from a quite realistic computer model simulating the acoustic interaction of $M$. elongata males in a chorus (Hartbauer 2008). Interestingly, follower signals in this simulation were shorter compared to leader signals although chirp plasticity was not implemented in this model, which simulates chorusing solely on realistic properties of song oscillators. It is therefore likely that signal plasticity originally evolved for energetic reasons in an ancestor species in which males exhibited a higher chirp rate.

\section{Energy saving in $M$. elongata}

A major factor rendering insect songs energetically inefficient arises from constraints due to an impedance mismatch between the sound-emitting radiator and the emitted wavelength (Prestwich 1994). Energy saving in acoustic interactions constitutes a strategy to combat an inefficient way of sound production common to almost all insects (for exception see burrowing crickets) where only a small fraction of energy invested in signal production is emitted as acoustic power (Prestwich 1994).

M. elongata males produce loud hemisyllables during wing opening and shorter, less intense hemisyllables during wing closing (Fig. 1c). This way of sound production is different from most ensiferans where loud song elements are commonly produced only during wing closing. Nevertheless, sound production during both wing cycles can be found in at least 13 European species of katydids (Heller 1988). In M. elongata syllable amplitude increases with wing stroke duration, wing velocity and maybe frictional force applied to the tegmen (Bailey and Broughton 1970; Montealegre-Z and Mason 2005). During synchronous entrainment, M. elongata males saved energy by a more efficient way of sound generation, achieved through a reduction of syllable number and a slight increase of wing stroke rate. Soft syllables at the onset of a chirp are the least efficient with respect to the related tegmen movement and the resulting acoustic output (Fig. 1d); a reduction of these soft syllables in chirps of a follower is therefore a more efficient way of chirp production.

$M$. elongata males saved energy during synchronous entrainment with signal rates faster than their intrinsic rate observed during solo singing. Theoretically, this "energy save mode" should allow males to signal for longer periods of time even at higher chirp rates. This hypothesis was investigated in experiments in which 10 males were entrained to three different signal rates $(1.8,2.0$ and $2.2 \mathrm{~s})$. However, the result was rather ambiguous, with only four males confirming the hypothesis, since they showed a higher singing activity when entrained to longer signal periods (blue lines in Fig. 7). Five males showed a similar total time spent singing per night irrespective of the entraining signal rate (red lines in Fig. 7). Surprisingly, one male showed the highest singing activity when entrained to the highest signal rate. Only two out of ten males spent a longer time singing at signal rates that corresponded to their solo chirp rates. The absence of a clear correlation between the total time spent singing with signal rate may be a consequence of an energetically cheap production of follower chirps. 


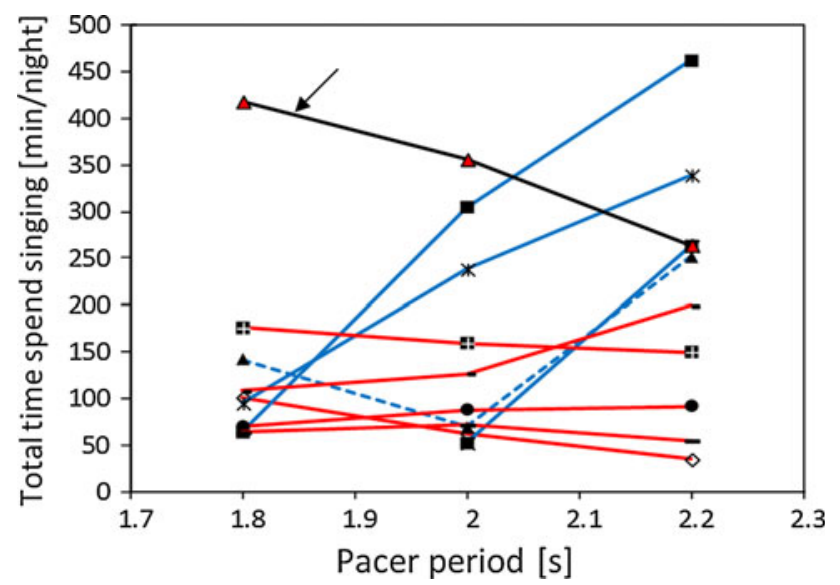

Fig. 7 Entrained singing activity. Total time spent singing during synchronous entrainment to three constant signal periods $(1.8,2.0$ and $2.2 \mathrm{~s})$, switched between successive nights $(N=10$ males, ambient temperature $27^{\circ} \mathrm{C}$ ). Arrow indicates a male with an extraordinary long solo chirp period of $2.7 \mathrm{~s}$

Further, indirect evidence for energy costs associated with signalling in Mecopoda was obtained via measurement of thoracic temperature. The major factors determining the cost of stridulation in singing crickets are wing stroke rate and the number of teeth struck per wing stroke (Prestwich and Walker 1981). Therefore, producing chirps is energetically less expensive than continuous trilling. For example, Oecanthus celerinictus and Oecanthus quadripunctatus show a 6-12 times higher metabolism associated with trilling compared to resting (Prestwich and Walker 1981). In the present study, the energetic difference between chirping and trilling was mirrored in a comparison of the thorax temperatures of three song variants of the genus M. elongata. Temperatures increased according to signalling effort and were highest in a trilling species (Fig. 6f). In Requena verticalis a wing stroke rate of only $9.7 \mathrm{~Hz}$ was insufficient to elevate thorax temperature significantly (Bailey et al. 1993). At the other extreme, a very fast wing stroke rate of $320-415 \mathrm{~Hz}$ in the bushcricket Hexacentrus unicolor increases thorax temperature $13-15^{\circ} \mathrm{C}$ above ambient temperature (Heller 1986).

An important result of the metabolic measurements is that singing at a high solo chirp rate is more costly (Fig. 6a). Since the likelihood for these males to become leader in acoustic interactions is higher (Hartbauer et al. 2005), females preferring leaders indirectly select males dealing with a higher energetic investment in their sexual display. However, in M. elongata solo chirp rate is not correlated with male quality, as indicated in nutrition experiments (Hartbauer et al. 2006). This suggests that a high solo chirp rate is less suitable as an honest indicator of male quality. A lack of correlation between males timing their signals in advance to others and male quality was also found in Hyla arborea (Richardson et al. 2008). Ongoing research aims to rule out whether
M. elongata males exhibit other properties like age, spermatophore size or fertility that correlate with solo chirp rate.

Phonotactic parasitoids

Probably more important than energy concerns are costs for signalling males due to the attraction of predators or parasitoids, which exploit the sexual signals of males (review in Zuk and Kolluru 1998). For crickets (Cade 1975; Zuk et al. 1998), katydids (Lehmann and Heller 1998) and cicadas (Lakes-Harlan et al. 2000) it has been shown that substantial costs for survival and reproduction can arise from these "unintended receivers". On a recent field trip in Malaysia, a collection of Mecopoda specimen revealed a number of individuals infected with larvae of a currently unknown parasitoid fly (Insecta, Diptera, Tachinidae). Members of this taxon are known for homing in on calling songs of katydids (Lehmann 2003). More intriguingly with respect to the differential signalling costs of leader and follower males, a recent study on the parasitoid fly Ormia ochracea (Diptera; Tachinidae) demonstrated that flies selectively approach the leading of two otherwise identical sound sources, while the lagging source had minimal influence on orientation (Lee et al. 2009). This opens up the fascinating possibility that these flies might have a preference for leading calls as well, similar to Mecopoda females, which in turn would impose a much stronger probability of parasitation for leader males compared to followers.

\section{Chirp plasticity favours signal jamming}

After perceiving a leader signal, males in a chorus will time their follower chirps in almost perfect synchrony (Hartbauer et al. 2005). Such signal jamming is even enhanced by the signal plasticity described in this study and will ultimately result in an increase of the signal-to-noise ratio of the chorus signal compared to a chirp of a single male, due to summation. This effect may be important for the maintenance of a conspecific chirp pattern in a noisy habitat like the nocturnal tropical rainforest. Indeed, Deily and Schul (2009) hypothesized that the unusually large amplitude modulation required for verse recognition in Neoconocephalus nebrascensis forces males to synchronize their calls in order to preserve an attractive call pattern for females. The same hypothesis has been tested for the synchronous flashing of fireflies (Photinus carolinus), with experimental evidence of a behavioural cooperative function of synchrony (Moiseff and Copeland 2010). Furthermore, an increased loudness of chorus signals may attract phonotactically responsive females from a greater distance. This suggests the high degree of signal overlap in $M$. elongata and $N$. nebrascensis as a cooperative act 
among males, rather than competition, a hypothesis with some support from female choice tests revealing a higher attractiveness of signals displayed in male aggregations, or duets compared to signals displayed in isolation (insects: Morris et al. 1978; Cade 1981; Doolan and Mac Nally 1981; Shelly and Greenfield 1991; Hyla microcephala: Schwartz 1994). An increased intensity of signals in aggregations was also necessary in order to simulate the evolution of chorus synchrony in an Indian Mecopoda species with a chirping call pattern, where females prefer leader males as well (Nityananda and Balakrishnan 2009).

Acknowledgments Funding was provided by the Austrian Science Foundation (FWF), project P 21808-B09 to M.H. We thank Brigitte Helfert for providing the trilling Mecopoda specimen from Thailand and Rosli Hashim for his generous help with field work in Malaysia.

Open Access This article is distributed under the terms of the Creative Commons Attribution Noncommercial License which permits any noncommercial use, distribution, and reproduction in any medium, provided the original author(s) and source are credited.

\section{References}

Arak A (1988) Female mate selection in the natterjack toad: active choice or passive attraction. Behav Ecol Sociobiol 22:317-327

Bailey WJ, Broughton WB (1970) The mechanics of stridulation in bush crickets (Tettigonioidea, Orthoptera). II. Conditions for resonance in the tegminal generator. J Exp Biol 52:505-517

Bailey WJ, Cunningham RR, Lebel L (1990) Song power, spectral distribution and female phonotaxis in the bushcricket Requena verticalis (Tettigoniidae: Orthoptera): active choice or passive attraction. Anim Behav 40:33-42

Bailey WJ, Withers PC, Endersby M, Gaull K (1993) The energetic costs of calling in the bushcricket Requena verticals (Orthoptera: Tettigoniidae: Listroscelidinae). J Exp Biol 178:21-37

Bennet-Clark HC (1970) The mechanisms and efficiency of sound production in mole crickets. J Exp Biol 52:619-652

Bennet-Clark HC (1971) Acoustics of insect sound. Nat Lond 234:255-259

Bennet-Clark HC (1975) Sound production in insects. Sci Prog Oxford 62:263-283

Bosch J, Márquez R (2002) Female preference function related to precedence effect in an amphibian anuran (Alytes cisternasii): tests with non-overlapping calls. Behav Ecol 13:149-153

Cade WH (1975) Acoustically orienting parasitoids: fly phonotaxis to cricket song. Science 190:1312-1313

Cade WH (1981) Field cricket spacing, and the phonotaxis of crickets and parasitoid flies to clumped and isolated cricket songs. Z Tierpsychol 55:365-375

Counter SA (1977) Bioacoustics and neurobiology of communication in the tettigoniid Neoconocephalus robustus. J Insect Physiol 23:993-1008

Dadour IR (1989) Temporal pattern changes in the calling song of the katydid Mygalopsis marki in response to conspecific song (Orthoptera: Tettigoniidae). J Insect Behav 2:199-215

Deily JA, Schul J (2009) Selective phonotaxis in Neoconocephalus nebrascensis (Orthoptera: Tettigoniidae): call recognition at two temporal scales. J Comp Physiol A 195:31-37
Doolan JM, Mac Nally RC (1981) Spatial dynamics and breeding ecology in the cicada Cystosoma saundersii: the interaction between distributions of resources and intraspecific behaviour. J Anim Ecol 50:925-940

Dyson ML, Passmore NI (1988) The combined effect of intensity and the temporal relationship of stimuli on phonotaxis in female painted reed frogs Hyperolius marmoratus. Anim Behav 36:1555-1556

Fertschai I, Stradner J, Römer H (2007) Neuroethology of female preference in the synchronously singing bushcricket Mecopoda elongata (Tettigoniidae; Orthoptera): why do followers call at all? J Exp Biol 210:465-476

Forrest TG (1991) Power output and efficiency of sound production by crickets. Behav Ecol 2:327-338

Forrest TG (1983) Calling songs and mate choice in mole crickets. In: Gwynne DT, Morris GK (eds) Orthopteran mating systems: sexual competition in a diverse group of insects. Westview Press, Boulder, pp 185-204

Galliart PL, Shaw KC (1996) The effect of variation in parameters of the male calling song of the katydid, Amblycorypha parvipennis (Orthoptera: Tettigoniidae), on female phonotaxis and phonoresponse. J Insect Behav 9:841-855

Gerhardt HC, Huber F (2002) Acoustic communication in insects and anurans: common problems and diverse solutions. University of Chicago Press, Chicago

Grafe TU (1996) The function of call alternation in the African reed frog (Hyperolius marmoratus): precise call timing prevents auditory masking. Behav Ecol Sociobiol 38:149-158

Greenfield MD (1994a) Cooperation and conflict in the evolution of signal interactions. Annu Rev Ecol Syst 25:97-126

Greenfield MD (1994b) Synchronous and alternating choruses in insects and anurans: common mechanisms and diverse functions. Am Zool 34:605-615

Greenfield MD, Minckley RL (1993) Acoustic dueling in tarbush grasshoppers: settlement of territorial contests via alternation of reliable signals. Ethology 95:309-326

Greenfield MD, Tourtellot MK, Snedden WA (1997) Precedence effects and the evolution of chorusing. Proc R Soc Lond B 264:1355-1361

Greenfield MD, Rand S (2000) Frogs have rules: selective attention algorithms regulate chorusing in Physalaemus pustulosus (Leptodactylidae). Ethology 106:331-347

Harrison JF (1989) Ventilatory frequency and haemolymph acid-base status during short-term hypercapnia in the locust, Schistocerca gregaria. J Insect Physiol 35:809-814

Hartbauer M (2008) Chorus model of the synchronizing bushcricket species Mecopoda elongata. Ecol Model 213:105-118

Hartbauer M, Kratzer S, Steiner K, Römer H (2005) Mechanisms for synchrony and alternation in song interactions of the bushcricket Mecopoda elongata (Tettigoniidae: Orthoptera). J Comp Physiol A 191:175-188

Hartbauer M, Kratzer S, Römer H (2006) Chirp rate is independent of male condition in a synchronising bushcricket. J Insect Physiol $52: 221-230$

Hedrick AV (1986) Female preferences for calling bout duration in a field cricket. Behav Ecol Sociobiol 19:73-77

Heller KG (1986) Warm-up and stridulation in the bushcricket, Hexacentrus unicolor serville (Orthoptera, Conocephalidae, Listroscelidinae). J Exp Biol 126:97-109

Heller KG (1988) Bioakustik der europäischen Laubheuschrecken. In: Knuth D (ed) Ökologie in Forschung und Anwendung. Verlag Josef Margraf, Weikersheim

Holzer B, Jacot A, Brinkhof MWG (2003) Condition-dependent signaling affects male sexual attractiveness in field crickets, Gryllus campestris. Behav Ecol 14:353-359

Howard RD, Palmer JG (1995) Female choice in Bufo americanus: effects of dominant frequency and call order. Copeia 1995:212-217 
Kavanagh MW (1987) The efficiency of sound production in two cricket species, Gryllotalpa australis and Teleogryllus commodus (Orthoptera: Grylloidea). J Exp Biol 130:107-119

Klump GM, Gerhardt HC (1992) Mechanisms and function of calltiming in male-male interactions in frogs. In: Mc Gregor PK (ed) Playback and studies of animal communication. Plenum Press, New York, pp 153-174

Kovac H, Stabentheiner A (1999) Effect of food quality on the body temperature of wasps (Paravespula vulgaris). J Insect Physiol 45:183-190

Kovac H, Stabentheiner A, Hetz SK, Petz M, Crailsheim K (2007) Respiration of resting honeybees. J Insect Physiol 53:1250-1261

Kovac H, Stabentheiner A, Schmaranzer S (2010) Thermoregulation of water foraging honeybees-balancing of endothermic activity with radiative heat gain and functional requirements. J Insect Physiol 56:1834-1845

Lakes-Harlan R, Stolting H, Moore TE (2000) Phonotactic behaviour of a parasitoid fly (Emblemasoma auditrix, Diptera, Sarcophagidae) in response to the calling song of its host Cicada (Okanagana rimosa, Homoptera, Cicadidae). Zoology 103:31-39

Latimer W (1981) The acoustic behaviour of Platycleis albopunctata (Goeze) (Orthoptera, Tettigoniidae). Behaviour 76:182-206

Lee N, Elias DO, Mason AC (2009) A precedence effect resolves phantom sound source illusions in the parasitoid fly Ormia ochracea. Proc Natl Acad Sci USA 106:6357-6362

Lehmann GUC (2003) Review of biogeography, host range and evolution of acoustic hunting in Ormiini (Insecta, Diptera, Tachinidae), parasitoids of night-calling bushcrickets and crickets (Insecta, Orthoptera, Ensifera). Zool Anz 242:107-120

Lehmann GUC, Heller KG (1998) Bushcricket song structure and predation by the acoustically orienting parasitoid fly Therobia leonidei (Diptera: Tachinidae: Ormiini). Behav Ecol Sociobiol 43:239-245

Loftus-Hills JJ (1974) Analysis of an acoustic pacemaker in Strecker's chorus frog, Pseudacris streckeri (Anura: Hylidae). J Comp Physiol 90:75-87

MacNally R, Young D (1981) Song energetics of the bladder cicada Cystosoma saundersii. J Exp Biol 90:185-196

Martínez-Rivera CC, Gerhardt HC (2008) Advertisement-call modification, male competition, and female preference in the birdvoiced treefrog Hyla avivoca. Behav Ecol Soc Biol 63:195-208

McLister JD (2001) Physical factors affecting the cost and efficiency of sound production in the treefrog Hyla versicolor. J Exp Biol 204:69-80

Minckley RL, Greenfield MD (1995) Psychoacoustics of female phonotaxis and the evolution of male signal interactions in Orthoptera. Ethol Ecol Evol 7:235-243

Moiseff A, Copeland J (2010) Firefly synchrony: a behavioral strategy to minimize visual clutter. Science 329:181

Montealegre-Z F, Mason AC (2005) The mechanics of sound production in Panacanthus pallicornis (Orthoptera: Tettigoniidae: Conocephalinae): the stridulatory motor patterns. J Exp Biol 208:1219-1237

Morris GK, Kerr G, Fullard JH (1978) Phonotactic preferences of female meadow katydids (Orthoptera: Tettigoniidae: Conocephalus nigropieurum). Can J Zool 56:1479-1487

Morris GK, DeLuca PA, Norton M, Mason A (2002) Calling-song function in male haglids (Orthoptera: Haglidae, Cyphoderris). Can J Zool 80:271-285

Nityananda V, Balakrishnan R (2009) Modeling the role of competition and cooperation in the evolution of katydid acoustic synchrony. Behav Ecol 20:484-489

Prestwich KN (1994) The energetics of acoustic signalling in anurans and insects. Am Zool 34:625-643

Prestwich KN, O'Sullivan K (2005) Simultaneous measurement of metabolic and acoustic power and the efficiency of sound production in two mole cricket species. J Exp Biol 208:1495-1512
Prestwich KN, Walker TJ (1981) Energetics of singing in crickets: effect of temperature in three trilling species (Orthoptera: Gryllidae). J Comp Physiol 143:199-212

Rand AS, Ryan MJ (1982) The adaptive significance of a complex vocal repertoire in a neotropical frog. Z Tierpsychol 57:209-214

Reinhold K, Greenfield MD, Jang Y, Broce A (1998) Energetic cost of sexual attractiveness: ultrasonic advertisement in wax moths. Anim Behav 55:905-913

Richardson C, Lena J-P, Joly P, Lengagne T (2008) Are leaders good mates? A study of call timing and male quality in a chorus situation. Anim Behav 76:1487-1495

Robinson DJ, Hall MJ (2002) Sound signalling in Orthoptera. Adv Insect Physiol 29:151-278

Ryan MJ (1986) Synchronized calling in a treefrog (Smilisca sila). Brain Behav Evol 29:196-206

Ryan MJ, Bartholomew WGA, Rand AS (1983) Energetics of reproduction in a neotropical frog, Physalaemus pustulosus. Ecology 64:1456-1462

Schwartz JJ (1994) Male advertisement and female choice in frogs: recent findings and new approaches to the study of communication in a dynamic acoustic environment. Am Zool 34:616-624

Schwartz JJ, Buchanan B, Gerhardt H (2002) Acoustic interactions among male gray treefrogs, Hyla versicolor, in a chorus setting. Behav Ecol Sociobiol 53:9-19

Shaw KC (1968) An analysis of the phonoresponse of males of the true katydid Pterophylla camellifolla (Fabricius) (Orthoptera: Tettigoniidae). Behaviour 31:203-260

Shelly TE, Greenfield MD (1991) Dominions and desert clickers (Orthoptera: Acrididae): influences of resources and male signaling on female settlement patterns. Behav Ecol Sociobiol 28:133-140

Sismondo E (1990) Synchronous, alternating, and phase-locked stridulation by a tropical katydid. Science $249: 55-58$

Snedden WA, Greenfield MD (1998) Females prefer leading males: relative call timing and sexual selection in katydid choruses. Anim Behav 56:1091-1098

Stabentheiner A, Schmaranzer S (1987) Thermographic determination of body temperatures in honey bees and hornets: Calibration and applications. Thermology 2:563-572

Stabentheiner A, Kovac H, Brodschneider R (2010) Honeybee colony thermoregulation-regulatory mechanisms and contribution of individuals in dependence on age, location and thermal stress. PLos One 5(e8967):1-13

Stevens ED, Josephson BK (1977) Metabolic rate and body temperature in singing katydids. Physiol Zool 50:31-42

Tauber E, Cohen D, Greenfield MD, Pener MP (2001) Duet singing and female choice in the bushcricket Phaneroptera nana. Behaviour 138:411-430

Tejedo M (1993) Do male natterjack toads join larger breeding choruses to increase mating success? Copeia 1993:75-80

Wagner WE Jr, Hoback WW (1999) Nutritional effects on male calling behaviour in the variable field cricket. Anim Behav 57:89-95

Walker TJ (1969) Acoustic synchrony: two mechanisms in the snowy tree cricket. Science 166:891-894

Wells KD, Taigen TL (1986) The effect of social interactions on calling energetics in the gray treefrog (Hyla versicolor). Behav Ecol Sociobiol 19:9-18

Whitney CL, Krebs JR (1975) Mate selection in Pacific Treefrogs. Nature 255:325-326

Zahavi A (1975) Mate selection: a selection for a handicap. J Theor Biol 53:205-214

Zuk M, Kolluru GR (1998) Exploitation of sexual signals by predators and parasitoids. Q Rev Biol 73:415-438

Zuk M, Rotenberry JT, Simmons LW (1998) Calling songs of field crickets (Teleogryllus oceanicus) with and without phonotactic parasitoid infection. Evolution 52:166-171 